\title{
Can We Execute Reliable MM-PBSA Free Energy Computations of Relative Stabilities of Different Guanine Quadruplex Folds?
}

\author{
Barira Islam, ${ }^{\dagger}$ Petr Stadlbauer, ${ }^{\dagger}$ Stephen Neidle, ${ }^{\S}$ Shozeb Haider ${ }^{\S}$ and Jiri Sponer ${ }^{\dagger \ddagger *}$ \\ ${ }^{\dagger}$ Institute of Biophysics, Academy of Sciences of the Czech Republic, Královopolská 135, 61265 \\ Brno, Czech Republic
}

${ }^{\S}$ UCL School of Pharmacy, 29-39 Brunswick Square, London WC1N 1AX, UK

${ }^{\ddagger}$ CEITEC - Central European Institute of Technology, Masaryk University, Campus Bohunice, Kamenice 5, 62500 Brno, Czech Republic

*Corresponding Author 
Equilibration protocol. The solvated system was first minimized (500 cycles of steepest descent cycles followed by 500 cycles of conjugate gradient cycles) with a restraint of $25 \mathrm{kcal} \mathrm{mol}^{-1} \AA^{-2}$ on the solute (including the channel cations). Minimization with $5 \mathrm{kcal} \mathrm{mol}^{-1} \AA^{-2}$ restraints followed, using 500 steps of steepest descent method and 500 steps of conjugate gradient. The restraints of 5 $\mathrm{kcal} \mathrm{mol}^{-1} \AA^{-2}$ were maintained on solute and the system was equilibrated for $50 \mathrm{ps}$ at constant temperature of $300 \mathrm{~K}$ and pressure of $1 \mathrm{~atm}$. An analogous series of alternating minimizations and equilibrations followed using decreasing position restraints of 4, 3, 2 and $1 \mathrm{kcal} \mathrm{mol}^{-1} \AA^{-2}$ consecutively. The final equilibration was carried out with position restraints of $0.5 \mathrm{kcal} \mathrm{mol}^{-1} \AA^{-2}$ and starting velocities from the previous equilibration, followed by a short free molecular dynamics simulation of 50 ps. Temperature and pressure coupling during equilibration was set to 0.2 and coupling during the last molecular dynamics phase was set to 5 .

Free energy calculations based on simulations of GSs of models 0-7. The RMSD variations of the GSs in the simulations without the loops showed slightly different trends from RMSDs in equivalent simulations of full GQs (Figures S4-S6). Contrary to the simulations with the loops, GS 5 displayed stable RMSD in the simulations without the loops in all the three force-fields. This further supported our justification that significantly higher RMSD values of model 5 in simulations of full GQs were due to stem-loop interactions as discussed in the Main text (Figures 10, S2 and S3). The RMSD values of GS 3 were unstable in bsc0 force field (Figure S4). Similarly, the RMSD values of GS 4 and GS 6 were unstable in the bsc0 $\chi_{\mathrm{OL} 4}$ force field (Figure S5). The significant fluctuations in RMSD values of GS 3, GS 4 and GS 6 reflected instabilities of these GSs in the simulations. The instabilities were further confirmed by visualization of trajectories as strand slippage was observed within few ns of the start of these simulations. In the simulations of GS $\mathbf{3}$ in bsc0 force field, the first guanine moved towards the bulk at 37 ns and then stacked over on the 5'-base of strand 3 at $~ 97$ ns. 
In the simulations of GS 4 and GS $\mathbf{6}$ in bsc $0 \chi_{\mathrm{OL} 4}$ force field, strand slippage was observed in the strand 4. For these geometries, we repeated the simulations with random seeds but could not achieve stable trajectories even after several attempts. It should be noted that, because the simulations correspond to non-native (experimentally unobserved) syn-anti GS patterns, the simulation behavior can be correct, though it may also indicate strain in the modeled starting structures.

It is noteworthy that in the simulations of the GSs from the models not all the 5'-syn bases could form the 5'-OH - (G)N3 H-bond (Table S9). Thus, while the GSs extracted from experimental topologies always sampled the 5'-OH - (G)N3 H-bonds in all the strands with 5'-terminal syn throughout the simulations in all the force-fields, the built-up models were not always capable to form them. It shows that the modeled starting structures are likely strained.

The MM-PBSA free energy results of model GSs are presented in the Tables S9 and S10. The energy of GS 5 was more converged than in the simulations with the loops. It showed a relative free energy of 3,2 and $-5 \mathrm{kcal} / \mathrm{mol}$ in the bsc0, bsc $0 \chi_{\mathrm{OL} 4}$ and $\mathrm{bsc} 0 \chi_{\mathrm{OL}} 4 \varepsilon \zeta_{\mathrm{OL} 1}$ simulations, respectively. GS 5 has 5'-syn in two strands and its base-steps are equivalent to GS $\mathbf{0}$. However, in the simulations of GS 5, the intramolecular hydrogen bond was formed only in strand 1 (Table S9).

The GS 2 and GS 4 have 5'-syn in two out of four strands and have equivalent base-steps. In the simulations, while both the strands of GS 2 sampled the intramolecular hydrogen bonds, these bonds were not formed in GS $\mathbf{4}$. Therefore, the predicted energy of GS $\mathbf{4}$ (by Cang et al. ${ }^{1}$ data) is higher than that of GS 2. GS 4 was highly structurally unstable in simulations in all three force-fields. In the simulations of GS 4, anti-syn transitions were observed in first base of strand 4 in the bsc0 and bsc $0 \chi_{\mathrm{OL} 4} \varepsilon \zeta_{\mathrm{OL} 1}$ force fields and strand slippage was observed in the strand (strand 4 ) in bsc $0 \chi_{\mathrm{OL} 4}$ force field. GS $\mathbf{3}$ and GS $\mathbf{6}$ are predicted to have highest energy among the models using the Cang et 
al.'s ${ }^{1}$ prediction. In agreement with this, the two model GSs showed higher energy than the other model GSs in the present MM-PBSA calculations (Table S9). GS 3 showed movement of 5’guanine from the first strand towards the bulk in the bsc0 force field. It had relative free energy of 23 and $15 \mathrm{kcal} / \mathrm{mol}$ in the more stable simulations with $\mathrm{bsc} 0 \chi_{\mathrm{OL} 4}$ and $\mathrm{bsc} 0 \chi_{\mathrm{OL} 4} \varepsilon \zeta_{\mathrm{OL} 1}$ force fields, respectively. GS 6 also showed high relative free energy in all the three force-fields. Thus, although, the energies in the three quartet system were more sensitive to minor structural perturbations, the trend of base-step stability is in agreement with the previous predictions based on two-quartet systems. ${ }^{1}$ The MM-GBSA values of the model GQs in simulations both with and without the loops also displayed similar trends (Table S11).

Legend to Supplementary Movie S1. The ion dynamics in Htel parallel-stranded GQ in the simulation carried out in the presence of $0.15 \mathrm{M}$ excess $\mathrm{NaCl}$ in $\operatorname{bsc} 0 \chi_{\mathrm{OL} 4} \varepsilon \zeta_{\mathrm{OL} 1}$ force field. The GQ is shown in cartoon representation. The backbone of full GQ is shown in cyan. The stem nucleosides are shown in grey and loop nucleosides are not shown. The ions between the quartets are visualized as purple and red spheres and the incoming bulk ion is shown as yellow sphere. The incoming ion is affecting the ions present in the channel, and the overall effect is that it reduces the energy barrier (activation energy) for departure of the channel ion on the opposite side of the GS. Due to the reasons explained in the main text, the non-polarizable force field is exaggerating the effective intercation repulsion and thus the probability of ion exchanges in simulations is likely exaggerated. The simulations show that the GS remains, in the course of the simulations, fully occupied by ions. 

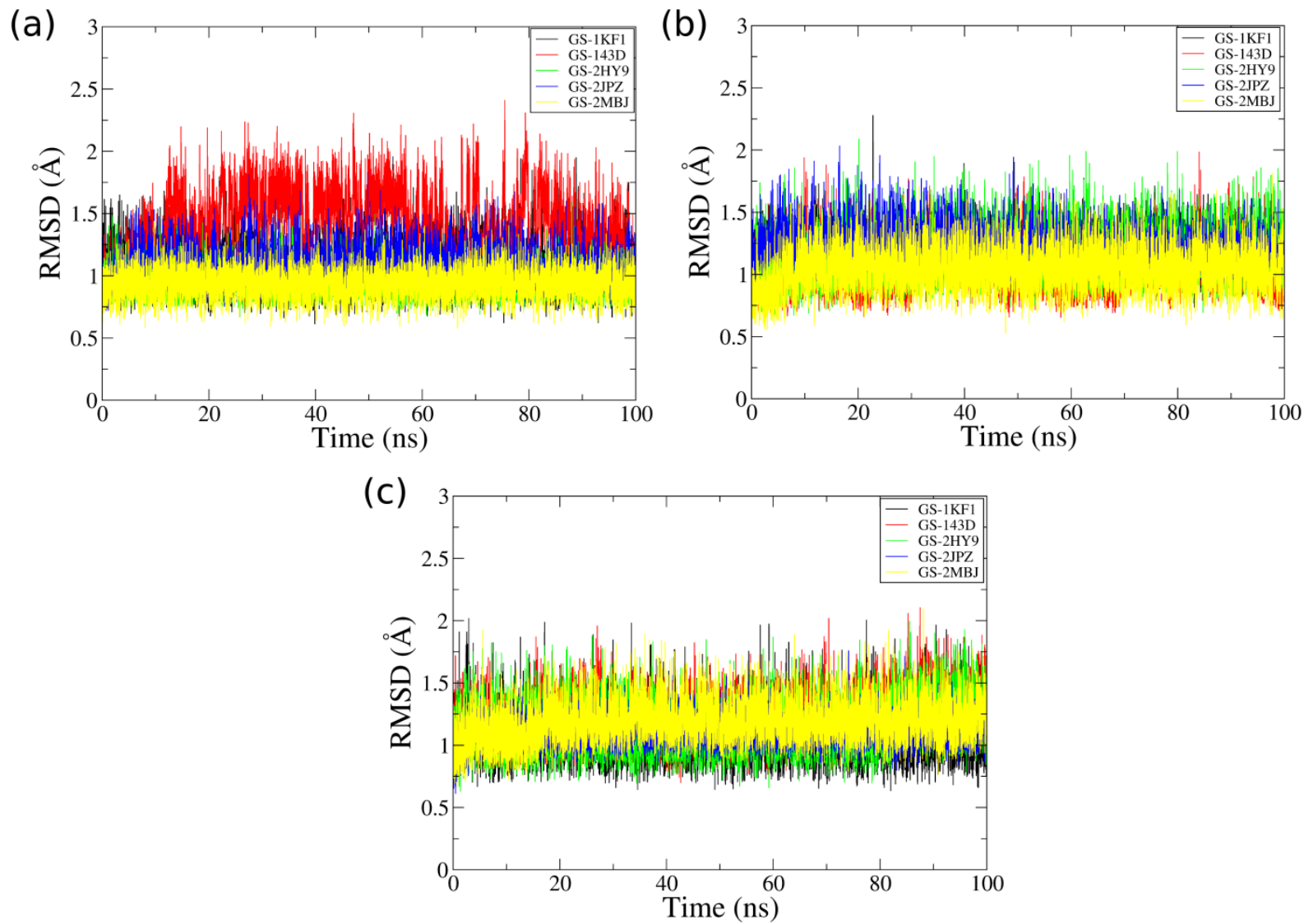

Figure S1. All atom mass-weighted RMSD vs. time plots for the Htel GSs simulated in (a) bsc0, (b) $\operatorname{bsc} 0 \chi_{\mathrm{OL} 4}$ and (c) bsc0$\chi_{\mathrm{OL} 4} \varepsilon \zeta_{\mathrm{OL} 1}$ force fields. The parallel-stranded (GS-1KF1), antiparallel basket (GS-143D), hybrid-1 (GS-2HY9), hybrid-2 (GS-2JPZ) and antiparallel (2+2) (GS-2MBJ) data are represented in black, red, green, blue and yellow. 


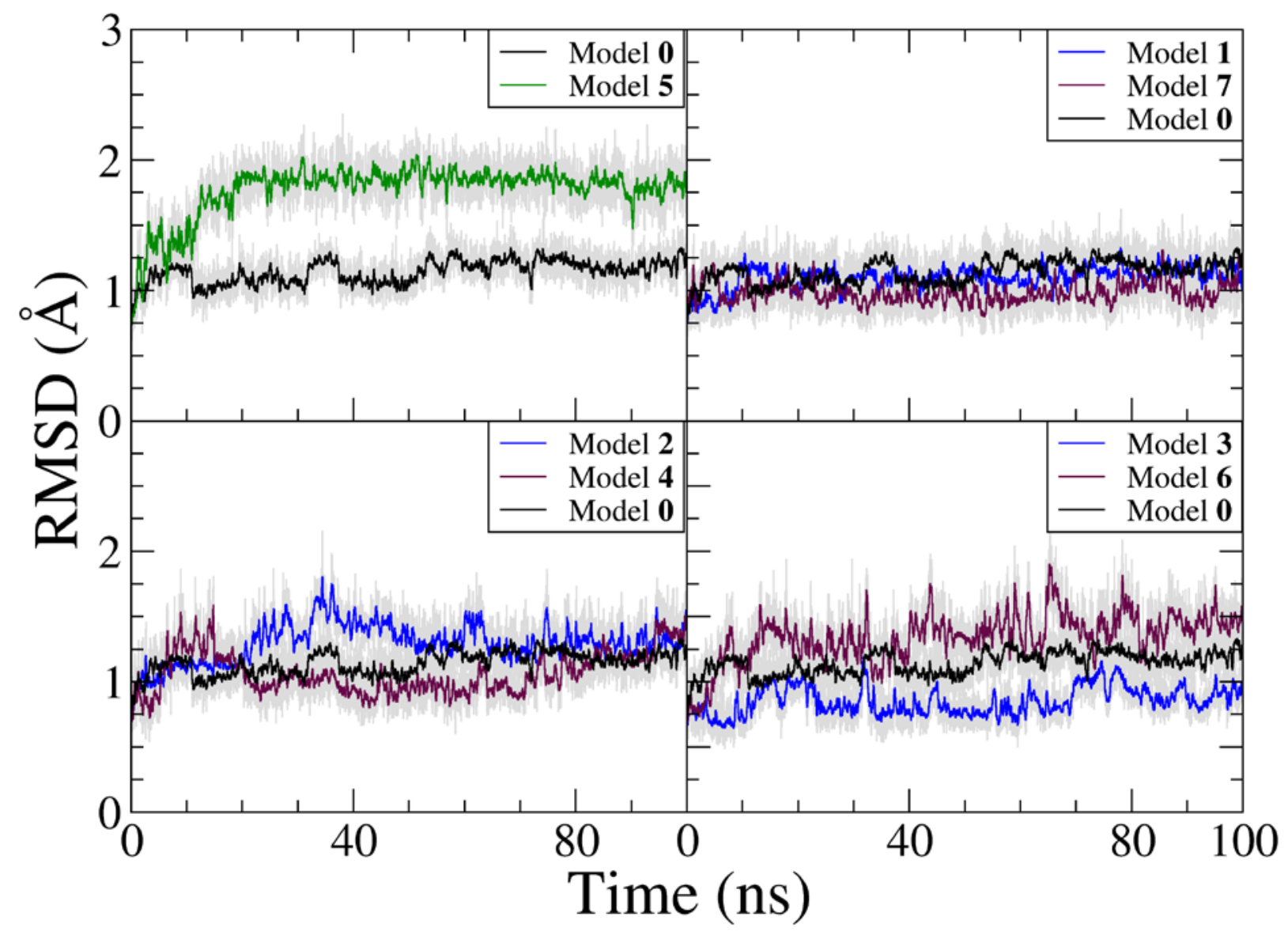

Figure S2. All atom mass-weighted RMSD vs. time plots for GSs of models $\mathbf{0}-\mathbf{7}$ in the simulations carried with the full GQs in the bsc0 force field. The grey lines in all the graphs represent the snapshots while the colored lines show the running averages over 250 ps. The models with identical base-steps are grouped together in the sub-plots. 


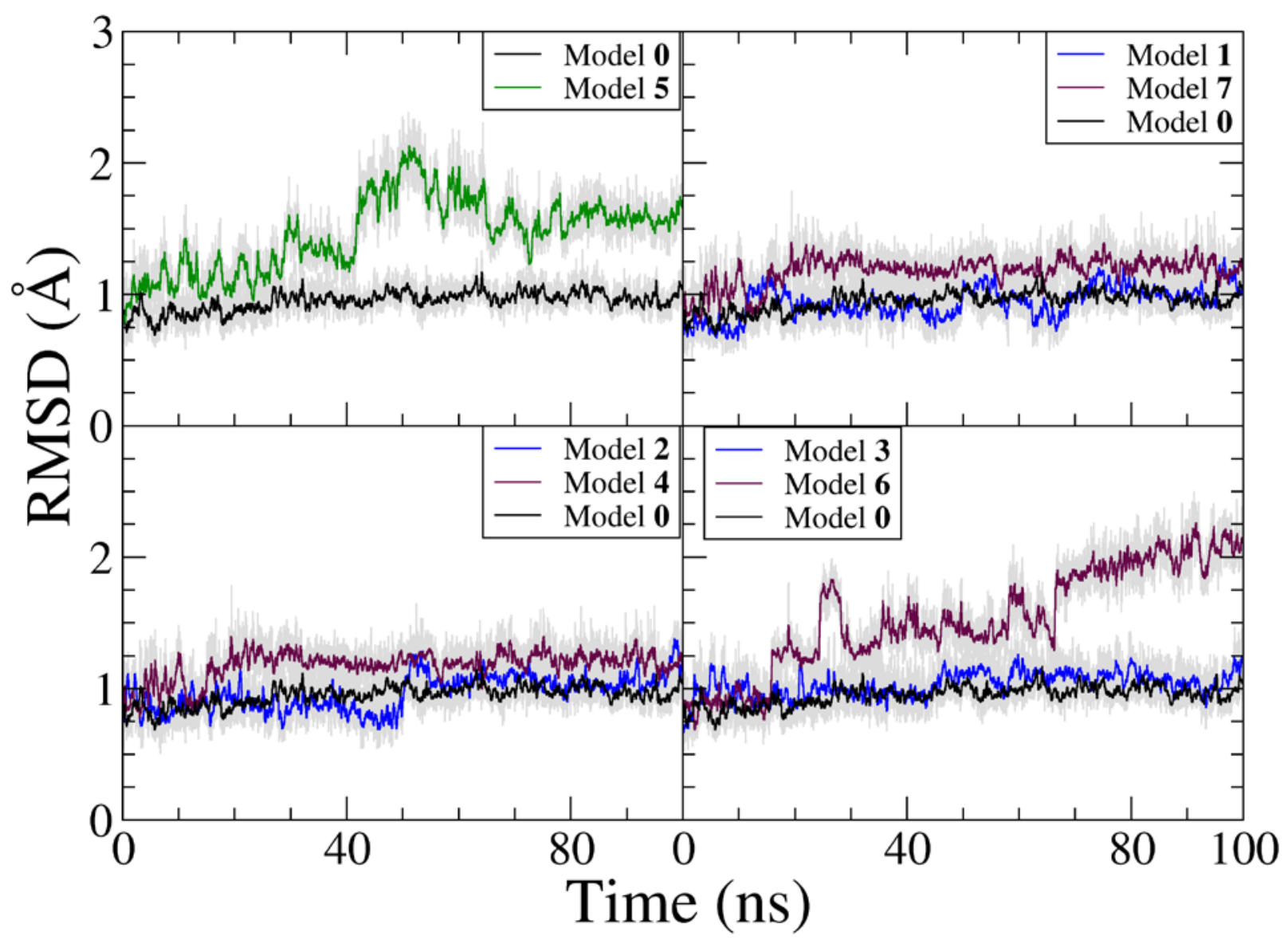

Figure S3. All atom mass-weighted RMSD vs. time plots for GSs of models in the simulations carried with the full GQ models in bsc $0 \chi_{\mathrm{OL} 4}$ force field. The grey lines in the graphs represent the snapshots while the colored lines show the running averages over 250 ps. The models with identical base-steps are grouped together in the sub-plots. 


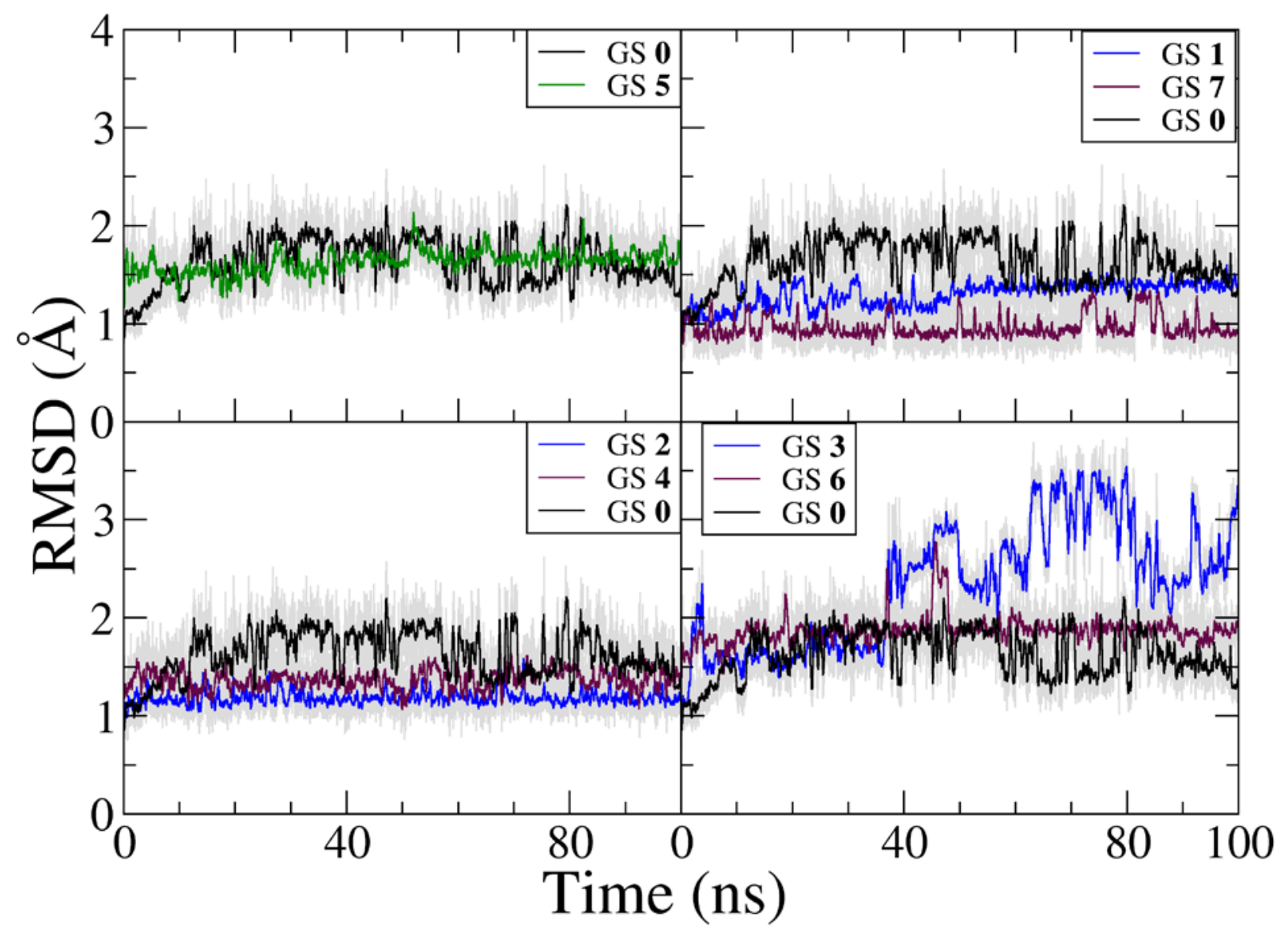

Figure S4. All atom mass-weighted RMSD vs. time plots for the GSs of the models in the bsc0 force field. The simulations were carried out with only the GSs. The grey lines in the graphs represent the snapshots while the colored lines show the running averages over 250 ps. The GSs with identical base-steps are grouped together in the sub-plots. 


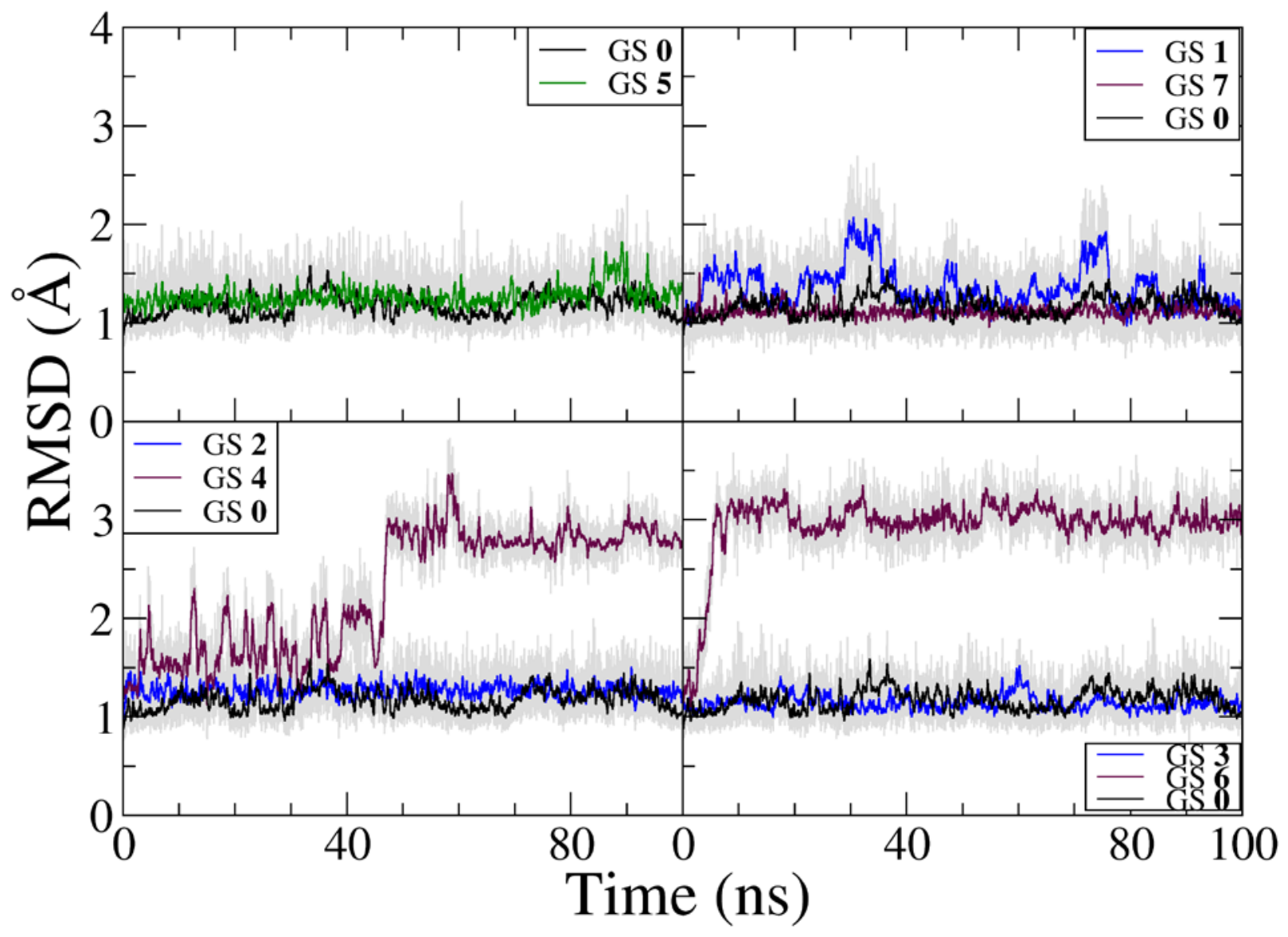

Figure S5. All atom mass-weighted RMSD vs. time plots for the GSs of the models in the bsc $0 \chi_{\mathrm{OL} 4}$ force field. The simulations were carried out with only the GSs. The grey lines in the graphs represent the snapshots while the colored lines show the running averages over 250 ps. The GSs with identical base-steps are grouped together in the sub-plots. 


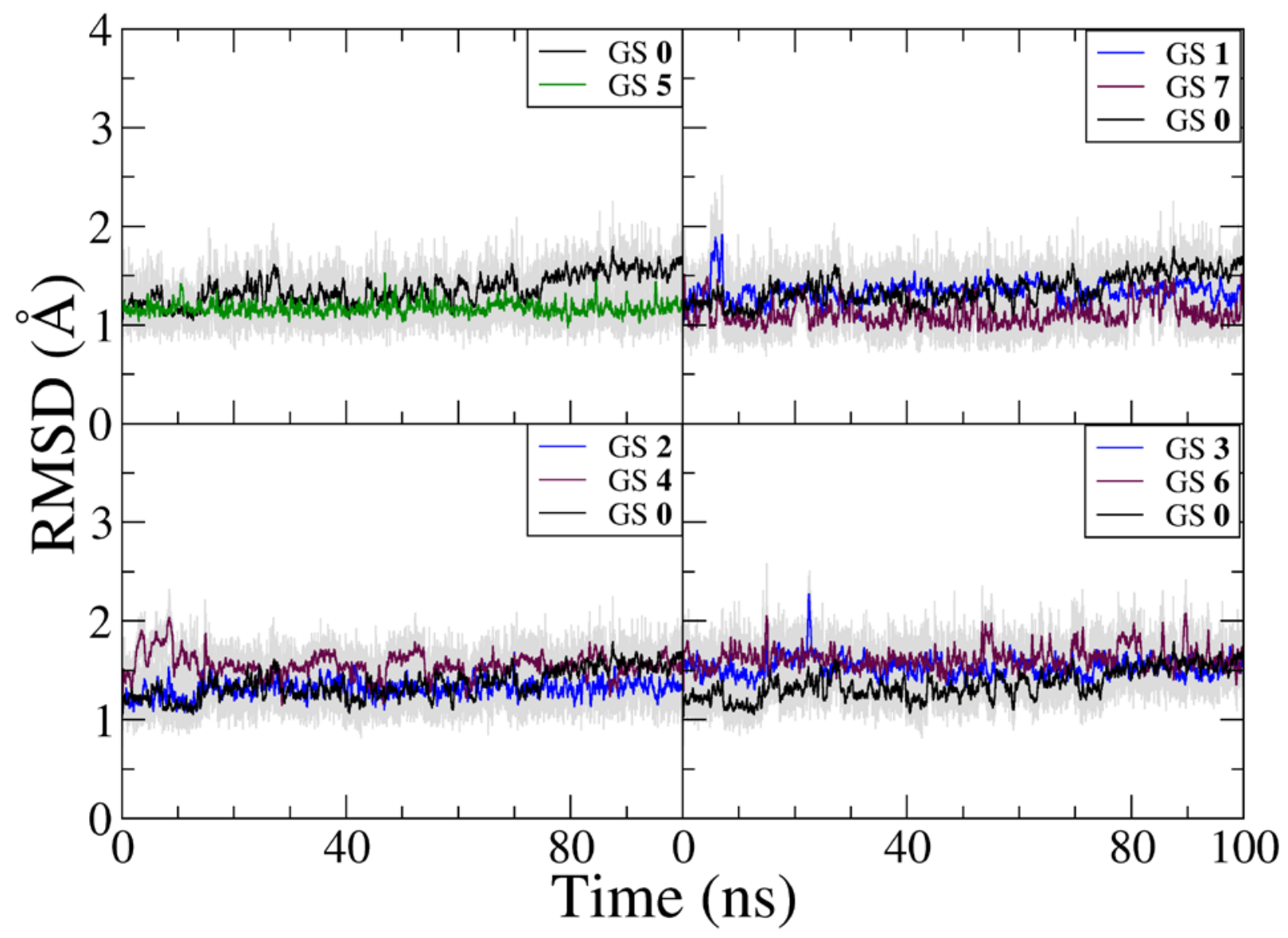

Figure S6. All atom mass-weighted RMSD vs. time plots for the GSs of the models in the $\operatorname{bsc} 0 \chi_{\mathrm{OL} 4} \varepsilon \zeta_{\mathrm{OL} 1}$ force field. The simulations were carried out with only the GSs. The grey lines in the graphs represent the snapshots while the colored lines show the running averages over 250 ps. The GSs with identical base-steps are grouped together in the sub-plots. 
Table S1: Glossary of Acronyms used in the Manuscript

\begin{tabular}{|l|l|}
\hline Acronym & Explanation of Acronym \\
\hline GQ & Guanine-Quadruplex \\
\hline GS & Guanine-Stem \\
\hline Htel & Human telomeric \\
\hline GpG & Guanine-Guanine dinucleotide step in quadruplex \\
\hline MM & Molecular Mechanics \\
\hline QM & Quantum Mechanics \\
\hline PBSA & Poisson Boltzmann Surface Area \\
\hline GBSA & Generalized Born Surface Area \\
\hline SASA & Solvent Accessible Surface Area \\
\hline PDB & Protein Data Bank \\
\hline RMSD & Root Mean Square Deviation \\
\hline APBS & Adaptive Poisson-Boltzmann Solver \\
\hline
\end{tabular}


Table S2. Summary of loop interactions and alignments involving flanking bases and loops formed in the Htel full GQ simulations. ${ }^{a}$

\begin{tabular}{|c|c|c|c|c|c|c|}
\hline \multirow[t]{2}{*}{ Topology } & \multicolumn{2}{|c|}{ bsc0 } & \multicolumn{2}{|c|}{$\operatorname{bsc} 0 \chi_{\mathrm{OL} 4}$} & \multicolumn{2}{|c|}{$\operatorname{bsc} 0 \chi_{\mathrm{OL} 4} \varepsilon \zeta_{\mathrm{OL} 1}$} \\
\hline & $\begin{array}{l}\text { Time } \\
\text { period } \\
\text { used for } \\
\text { MM- } \\
\text { PBSA } \\
\text { (ns) }\end{array}$ & $\begin{array}{l}\text { Remark } \\
\text { about } \\
\text { structures } \\
\text { seen in the } \\
\text { simulation }\end{array}$ & $\begin{array}{l}\text { Time } \\
\text { period } \\
\text { used } \\
\text { for } \\
\text { MM- } \\
\text { PBSA } \\
\text { (ns) }\end{array}$ & $\begin{array}{l}\text { Remark } \\
\text { about } \\
\text { structures } \\
\text { seen in the } \\
\text { simulation }\end{array}$ & $\begin{array}{l}\text { Time } \\
\text { period } \\
\text { used } \\
\text { for } \\
\text { MM- } \\
\text { PBSA } \\
\text { (ns) }\end{array}$ & $\begin{array}{l}\text { Remark } \\
\text { about } \\
\text { structures } \\
\text { seen in the } \\
\text { simulation }\end{array}$ \\
\hline $\begin{array}{l}\text { Parallel- } \\
\text { stranded }\end{array}$ & $50-100$ & $\begin{array}{l}\text { Above: nil } \\
\text { Groove: } \\
\text { GS-L in the } \\
\text { grooves } 1,2 \\
\text { and } 3 \\
\text { Below: nil }\end{array}$ & $50-100$ & $\begin{array}{l}\text { Above: nil } \\
\text { Groove: } \\
\text { GS-L in the } \\
\text { grooves } 1,2 \\
\text { and } 3 \\
\text { Below: nil }\end{array}$ & $50-100$ & $\begin{array}{l}\text { Above: nil } \\
\text { Groove: GS- } \\
\text { L in the } \\
\text { grooves } 1,2 \\
\text { and } 3 \\
\text { Below: nil } \\
\end{array}$ \\
\hline $\begin{array}{l}\text { Anti-parallel } \\
\text { basket }\end{array}$ & $50-100$ & $\begin{array}{l}\text { Above: S, } \\
\text { GS-L } \\
\text { Groove: nil } \\
\text { Below: T }\end{array}$ & $50-100$ & $\begin{array}{l}\text { Above: BP } \\
\text { Groove: nil } \\
\text { Below: BP }\end{array}$ & $50-100$ & $\begin{array}{l}\text { Above: BP } \\
\text { Groove: nil } \\
\text { Below: BP }\end{array}$ \\
\hline Hybrid-1 & $50-100$ & $\begin{array}{l}\text { Above: } \mathrm{S} \\
\text { Groove : } \\
\text { GS-L in the } \\
\text { groove } 1 \\
\text { Below: } \mathrm{T} \\
\end{array}$ & $50-100$ & $\begin{array}{l}\text { Above: S } \\
\text { Groove: } \\
\text { GS-L in } \\
\text { groove } 1 \\
\text { Below: BP }\end{array}$ & $50-100$ & $\begin{array}{l}\text { Above: } \mathrm{S}, \mathrm{BP} \\
\text { Groove: GS- } \\
\mathrm{L} \text { in the } \\
\text { groove } 1 \\
\text { Below: } \mathrm{T} \\
\end{array}$ \\
\hline Hybrid-2 & $50-100$ & $\begin{array}{l}\text { Above: } \mathrm{T}, \mathrm{S} \\
\text { Groove: } \\
\text { GS-L in the } \\
\text { groove } 3 \\
\text { Below: } \mathrm{T}\end{array}$ & $50-100$ & $\begin{array}{l}\text { Above: T, S } \\
\text { Groove: GS- } \\
\text { L in groove } \\
3 \\
\text { Below: BP }\end{array}$ & $50-100$ & $\begin{array}{l}\text { Above: T, S } \\
\text { Groove: GS-L } \\
\text { in the groove } \\
3 \\
\text { Below: BP, S }\end{array}$ \\
\hline $\begin{array}{l}\text { Anti-parallel } \\
(2+2)\end{array}$ & $50-100$ & $\begin{array}{l}\text { Above: T } \\
\text { Groove: } \\
\text { GS-L } \\
\text { Below: two } \\
\mathrm{T}\end{array}$ & $50-100$ & $\begin{array}{l}\text { Above-T } \\
\text { Groove: GS- } \\
\text { L } \\
\text { Below: T, } \\
\text { BP }\end{array}$ & $50-100$ & $\begin{array}{l}\text { Above-T } \\
\text { Groove: GS-L } \\
\text { Below: T, S }\end{array}$ \\
\hline
\end{tabular}

${ }^{a}$ The loops can align above the first quartet, below the third quartet and can interact in the grooves of the GQ. The Table summarizes these interactions, which may affect the conformations of the GSs and thus the MM-PBSA data. A number is specified when more than one interaction/alignment is formed, which is particularly relevant for the grooves. The loops can interact with the GSs to form stable hydrogen bonds (GS-L). Two or more flanking and/or loop nucleotides can stack over one another (S) to form ladder-like structure. The flanking and/or loop nucleotides can form hydrogen bonds and align to form triad (T) or base-pair (BP). 
Table S3. MM-PBSA-based free energies ${ }^{a}(\mathrm{kcal} / \mathrm{mol})$ of the Htel topologies. ${ }^{b}$

\begin{tabular}{|c|c|c|c|c|c|c|c|c|}
\hline Topology & MM_ele & MM_vdw & MM_int & PB_sur & E_disp & PB_cal & $G$ & $\Delta \boldsymbol{G}$ \\
\hline \multicolumn{9}{|l|}{ bsc0 } \\
\hline Parallel-stranded & -1461 & -106 & 559 & 27 & -258 & -1894 & -3133 & 0 \\
\hline Antiparallel basket & -1318 & -105 & 548 & 27 & -250 & -2014 & -3112 & 21 \\
\hline Hybrid-1 & -1399 & -110 & 560 & 27 & -249 & -1942 & -3113 & 20 \\
\hline Hybrid-2 & -1390 & -107 & 558 & 27 & -253 & -1957 & -3122 & 11 \\
\hline Antiparallel (2+2) & -1349 & -105 & 545 & 27 & -248 & -1998 & -3128 & 5 \\
\hline \multicolumn{9}{|l|}{ bsc0 $\chi_{\mathrm{OL} 4}$} \\
\hline Parallel-stranded & -1472 & -102 & 562 & 27 & -258 & -1888 & -3131 & 0 \\
\hline Antiparallel basket & -1364 & -103 & 553 & 27 & -251 & -1978 & -3116 & 15 \\
\hline Hybrid-1 & -1417 & -103 & 561 & 27 & -250 & -1931 & -3113 & 18 \\
\hline Hybrid-2 & -1408 & -103 & 562 & 27 & -252 & -1934 & -3108 & 23 \\
\hline Antiparallel $(2+2)$ & -1338 & -106 & 543 & 27 & -248 & -2001 & -3123 & 8 \\
\hline \multicolumn{9}{|l|}{$\operatorname{bsc} 0 \chi_{\mathrm{OL} 4} \varepsilon_{\zeta_{\mathrm{OL} 1}}$} \\
\hline Parallel-stranded & -1472 & -101 & 563 & 27 & -258 & -1887 & -3128 & 0 \\
\hline Antiparallel basket & -1340 & -104 & 548 & 27 & -250 & -1996 & -3115 & 13 \\
\hline Hybrid-1 & -1391 & -106 & 559 & 27 & -247 & -1951 & -3109 & 19 \\
\hline Hybrid-2 & -1407 & -104 & 559 & 27 & -250 & -1937 & -3112 & 16 \\
\hline Antiparallel (2+2) & -1358 & -106 & 548 & 27 & -248 & -1987 & -3124 & 4 \\
\hline
\end{tabular}

${ }^{a}$ The MM_ele, MM_vdw and MM_int represent the electrostatic, van der Waals and the internal (bond, angle and dihedral angle) potential energies. The PB_sur and PB_cal represent non-polar cavitation and polar solvation energies. E_disp represents the solute-solvent dispersion energy. $G$ is the summation of all the above terms and $\Delta G$ is the relative free energy calculated with respect to the parallel-stranded topology in the same force-field. All the values were rounded-off to the nearest whole numbers.

${ }^{b}$ The simulations were carried with the full GQ in $0.15 \mathrm{M}$ excess $\mathrm{NaCl}$. Only the GSs were used for MM-PBSA calculations. The 3'-terminal hydrogen was removed from parallel-stranded and antiparallel-basket GQ for MM-PBSA calculations to maintain equal number of atoms in all the GSs. 
Table S4. MM-PBSA-based free energies ${ }^{a}(\mathrm{kcal} / \mathrm{mol})$ of the GSs of Htel topologies ${ }^{b}$ in the simulations carried out in $0.15 \mathrm{M}$ excess $\mathrm{NaCl}$.

\begin{tabular}{|c|c|c|c|c|c|c|c|c|}
\hline GS-Topology & MM_ele & MM_vdw & MM_int & PB_sur & E_disp & PB_cal & $G$ & $\Delta G$ \\
\hline \multicolumn{9}{|l|}{ bsc0 } \\
\hline Parallel-stranded & -1993 & -105 & 552 & 25 & -233 & -999 & -2753 & 0 \\
\hline Antiparallel basket & -1957 & -99 & 537 & 25 & -234 & -1027 & -2755 & -2 \\
\hline Hybrid-1 & -2033 & -100 & 546 & 25 & -232 & -968 & -2762 & -9 \\
\hline Hybrid-2 & -2017 & -101 & 539 & 25 & -232 & -985 & -2771 & -18 \\
\hline Antiparallel (2+2) & -1974 & -102 & 526 & 25 & -230 & -1020 & -2775 & -22 \\
\hline \multicolumn{9}{|l|}{ bsc0 $\chi_{\text {OL4 }}$} \\
\hline Parallel-stranded & -1995 & -104 & 550 & 25 & -233 & -998 & -2755 & 0 \\
\hline Antiparallel basket & -1961 & -101 & 540 & 25 & -233 & -1029 & -2759 & -4 \\
\hline Hybrid-1 & -2013 & -101 & 542 & 25 & -232 & -988 & -2767 & -12 \\
\hline Hybrid-2 & -2022 & -101 & 541 & 25 & -231 & -982 & -2770 & -15 \\
\hline Antiparallel (2+2) & -1978 & -101 & 529 & 25 & -232 & -1020 & -2777 & -22 \\
\hline \multicolumn{9}{|l|}{ bsc0 $\chi_{O L 4} \varepsilon \zeta_{O L 1}$} \\
\hline Parallel-stranded & -2000 & -101 & 552 & 25 & -233 & -992 & -2749 & 0 \\
\hline Antiparallel basket & -1960 & -101 & 545 & 25 & -233 & -1025 & -2749 & 0 \\
\hline Hybrid-1 & -2031 & -101 & 549 & 25 & -233 & -971 & -2762 & -13 \\
\hline Hybrid-2 & -2010 & -101 & 539 & 25 & -231 & -989 & -2767 & -18 \\
\hline Antiparallel (2+2) & -2009 & -100 & 539 & 25 & -232 & -992 & -2769 & -20 \\
\hline
\end{tabular}

${ }^{a}$ The individual terms in the Table are explained in the footnotes of Table S3.

${ }^{b}$ Simulations and MM-PBSA calculations were carried out on the GSs of Htel topologies. 
Table S5. MM-PBSA-based free energies ${ }^{a}(\mathrm{kcal} / \mathrm{mol})$ of GSs of Htel topologies ${ }^{b}$ in the simulations carried out in $0.15 \mathrm{M}$ excess $\mathrm{KCl}$.

\begin{tabular}{|c|c|c|c|c|c|c|c|c|}
\hline GS-Topology & MM_ele & MM_vdw & MM_int & PB_sur & E_disp & PB_cal & $G$ & $\Delta G$ \\
\hline \multicolumn{9}{|l|}{ bsc0 } \\
\hline Parallel-stranded & -1967 & -100 & 553 & 26 & -234 & -995 & -2717 & 0 \\
\hline Antiparallel basket & -1913 & -99 & 540 & 25 & -233 & -1036 & -2716 & 1 \\
\hline Hybrid-1 & -1998 & -97 & 542 & 25 & -234 & -974 & -2736 & -19 \\
\hline Hybrid-2 & -1990 & -96 & 539 & 25 & -233 & -983 & -2738 & -21 \\
\hline Antiparallel (2+2) & -1948 & -98 & 527 & 25 & -231 & -1016 & -2741 & -24 \\
\hline \multicolumn{9}{|l|}{$\operatorname{bsc} 0 \chi_{\mathrm{oL} 4}$} \\
\hline Parallel-stranded & -1968 & -100 & 551 & 26 & -234 & -993 & -2718 & 0 \\
\hline Antiparallel basket & -1940 & -99 & 545 & 25 & -234 & -1019 & -2722 & -4 \\
\hline Hybrid-1 & -1997 & -97 & 548 & 25 & -235 & -975 & -2731 & -13 \\
\hline Hybrid-2 & -1969 & -99 & 537 & 25 & -234 & -998 & -2738 & -20 \\
\hline Antiparallel (2+2) & -1949 & -97 & 529 & 25 & -233 & -1016 & -2741 & -23 \\
\hline \multicolumn{9}{|l|}{ bsc0 $\chi_{\mathrm{OL} 4} \varepsilon \zeta_{\mathrm{OL} 1}$} \\
\hline Parallel-stranded & -1974 & -97 & 554 & 26 & -238 & -987 & -2716 & 0 \\
\hline Antiparallel basket & -1939 & -99 & 546 & 25 & -234 & -1019 & -2720 & -4 \\
\hline Hybrid-1 & -2003 & -98 & 549 & 25 & -235 & -965 & -2727 & -11 \\
\hline Hybrid-2 & -1975 & -99 & 539 & 25 & -233 & -990 & -2733 & -17 \\
\hline Antiparallel (2+2) & -1954 & -98 & 531 & 25 & -232 & -1009 & -2737 & -21 \\
\hline
\end{tabular}

${ }^{a}$ The individual terms in the Table are explained in the footnotes of Table S3.

${ }^{b}$ Simulations and MM-PBSA calculations were carried out on the GSs of Htel topologies.

Note that the overall trend of energy variation among the topologies in GSs is similar to that observed in the simulations carried out in $\mathrm{Na}^{+}$ions (Table 1 (simulations without the loops, i.e., GSs only simulations) and Table S4). 
Table S6. MM-GBSA-based free energies (kcal/mol) of the Htel topologies in the simulations carried out in $0.15 \mathrm{M}$ excess $\mathrm{NaCl}$.

\begin{tabular}{|c|c|c|c|c|}
\hline Topology & $G^{a}$ & $\Delta G^{a}$ & $G^{b}$ & $\Delta G^{b}$ \\
\hline \multicolumn{5}{|l|}{ bsc0 } \\
\hline Parallel-stranded & -2721 & 0 & -2451 & 0 \\
\hline Antiparallel-basket & -2717 & 4 & -2447 & 4 \\
\hline Hybrid-1 & -2715 & 6 & -2452 & -1 \\
\hline Hybrid-2 & -2715 & 6 & -2459 & -8 \\
\hline Antiparallel (2+2) & -2725 & -4 & -2466 & -15 \\
\hline \multicolumn{5}{|l|}{$\operatorname{bsc} 0 \chi_{\mathrm{OL} 4}$} \\
\hline Parallel-stranded & -2720 & 0 & -2452 & 0 \\
\hline Antiparallel-basket & -2716 & 4 & -2449 & 2 \\
\hline Hybrid-1 & -2718 & 2 & -2457 & -5 \\
\hline Hybrid-2 & -2705 & 15 & -2458 & -6 \\
\hline Antiparallel (2+2) & -2726 & -6 & -2463 & -11 \\
\hline \multicolumn{5}{|l|}{ bsc0 $\chi_{\mathrm{OL} 4} \varepsilon \zeta_{\mathrm{OL} 1}$} \\
\hline Parallel-stranded & -2721 & 0 & -2447 & 0 \\
\hline Antiparallel-basket & -2719 & 2 & -2441 & 6 \\
\hline Hybrid-1 & -2718 & 3 & -2452 & -5 \\
\hline Hybrid-2 & -2717 & 4 & -2459 & -12 \\
\hline Antiparallel (2+2) & -2726 & -5 & -2460 & -13 \\
\hline
\end{tabular}

${ }^{a}$ Simulations carried out with full GQ and subsequent MM-GBSA carried out with GSs.

${ }^{b}$ Simulations and MM-GBSA calculations carried out with the GSs only.

$G$ represents the energy of the topology while $\Delta G$ represents relative free energy with respect to parallel-stranded GQ in the same force-field. 
Table S7. Relative free energies (kcal/mol) of Htel GS topologies in three independent sets of 100 ns long simulations with full GQ. ${ }^{a}$

\begin{tabular}{|c|c|c|c|}
\hline Topology & Relative free energy $^{b}$ & Relative free energy ${ }^{c}$ & Relative free energy ${ }^{d}$ \\
\hline \multicolumn{4}{|l|}{ bsc0 } \\
\hline Parallel stranded & 0 & 0 & 0 \\
\hline Antiparallel basket & $\begin{array}{l}21 \\
\text { Stem-loop interaction }\end{array}$ & 16 & 10 \\
\hline Hybrid-1 & 20 & 17 & 15 \\
\hline Hybrid-2 & 11 & 12 & 20 \\
\hline Antiparallel (2+2) & 5 & 13 & 12 \\
\hline \multicolumn{4}{|l|}{ bsc0 $\chi_{\mathrm{OL} 4}$} \\
\hline Parallel stranded & 0 & 0 & 0 \\
\hline Antiparallel basket & 15 & 14 & 13 \\
\hline Hybrid-1 & 18 & 12 & 18 \\
\hline Hybrid-2 & 23 & 17 & 18 \\
\hline Antiparallel $(2+2)$ & 8 & $\begin{array}{l}23 \\
\text { cation distance } \sim 6 \AA\end{array}$ & 7 \\
\hline \multicolumn{4}{|l|}{ bsc0 $\chi_{\mathrm{OL} 4} \varepsilon \zeta_{\mathrm{OL} 1}$} \\
\hline Parallel stranded & 0 & 0 & 0 \\
\hline Antiparallel basket & 13 & 19 & $\begin{array}{l}23 \\
\text { Stem-loop interaction }\end{array}$ \\
\hline Hybrid-1 & 19 & 16 & 14 \\
\hline Hybrid-2 & 16 & 10 & 15 \\
\hline Antiparallel (2+2) & 4 & 3 & 3 \\
\hline
\end{tabular}

${ }^{a}$ The relative free energy of the topologies showed significant variations even within the same forcefield. In topologies showing a difference of $\geq 10 \mathrm{kcal} / \mathrm{mol}$ from the lowest of the three values in the same force-field, the probable reason for deviation is noted below the relative free energy of the respective simulation.

${ }^{b, c, d}$ Relative free energies calculated from three independent MD simulations carried out in 0.15 M excess $\mathrm{NaCl}$. The simulations were carried out with the full GQ and subsequent MM-PBSA calculations were carried out only on GSs. 
Table S8. MM-PBSA-based free energies ${ }^{a}(\mathrm{kcal} / \mathrm{mol})$ of the model GQs. ${ }^{b}$

\begin{tabular}{|c|c|c|c|c|c|c|c|c|}
\hline Model & MM_ele & MM_vdw & MM_int & PB_sur & E_disper & PB_cal & $G$ & $\Delta \mathbf{G}$ \\
\hline \multicolumn{9}{|l|}{ bsc0 } \\
\hline 0 & -1380 & -104 & 549 & 27 & -250 & -1935 & -3093 & 0 \\
\hline 1 & -1406 & -100 & 557 & 27 & -255 & -1917 & -3094 & -1 \\
\hline 2 & -1358 & -108 & 547 & 27 & -252 & -1946 & -3090 & 3 \\
\hline 3 & -1392 & -99 & 553 & 27 & -254 & -1920 & -3085 & 8 \\
\hline 4 & -1426 & -100 & 557 & 27 & -252 & -1895 & -3089 & 4 \\
\hline 5 & -1394 & -95 & 562 & 27 & -259 & -1924 & -3083 & 10 \\
\hline 6 & -1376 & -99 & 559 & 27 & -253 & -1931 & -3073 & 20 \\
\hline 7 & -1452 & -99 & 561 & 27 & -256 & -1886 & -3105 & -12 \\
\hline \multicolumn{9}{|c|}{ bsc0 $\chi_{\text {oL4 }}$} \\
\hline $\mathbf{0}$ & -1424 & -103 & 554 & 27 & -252 & -1900 & -3098 & 0 \\
\hline 1 & -1477 & -103 & 561 & 27 & -254 & -1862 & -3108 & -10 \\
\hline 2 & -1427 & -105 & 563 & 27 & -252 & -1891 & -3085 & 13 \\
\hline 3 & -1415 & -102 & 552 & 27 & -252 & -1904 & -3094 & 4 \\
\hline 4 & -1466 & -99 & 561 & 27 & -254 & -1861 & -3092 & 6 \\
\hline 5 & -1486 & -95 & 565 & 27 & -257 & -1853 & -3099 & -1 \\
\hline 6 & -1433 & -101 & 569 & 27 & -253 & -1889 & -3080 & 18 \\
\hline 7 & -1491 & -100 & 566 & 27 & -255 & -1854 & -3107 & -9 \\
\hline \multicolumn{9}{|c|}{$\operatorname{bsc} 0 \chi_{\mathrm{OL} 4} \varepsilon \zeta_{\mathrm{OL} 1}$} \\
\hline $\mathbf{0}$ & -1399 & -104 & 549 & 27 & -250 & -1919 & -3096 & 0 \\
\hline 1 & -1484 & -101 & 561 & 27 & -253 & -1849 & -3099 & -3 \\
\hline 2 & -1379 & -110 & 549 & 27 & -249 & -1932 & -3094 & 2 \\
\hline 3 & -1397 & -106 & 556 & 27 & -250 & -1912 & -3082 & 14 \\
\hline 4 & -1466 & -101 & 563 & 27 & -253 & -1860 & -3090 & 6 \\
\hline 5 & -1489 & -100 & 572 & 27 & -252 & -1846 & -3088 & 8 \\
\hline 6 & -1433 & -101 & 568 & 27 & -253 & -1884 & -3076 & 20 \\
\hline 7 & -1484 & -102 & 555 & 27 & -251 & -1848 & -3103 & -7 \\
\hline
\end{tabular}

${ }^{a}$ The individual terms in the Table are explained in the footnotes of the Table S3.

${ }^{b}$ Simulations were carried with the full GQs but only GSs were used for the MM-PBSA calculations. The simulations were carried out in $0.15 \mathrm{M}$ excess $\mathrm{NaCl}$. 


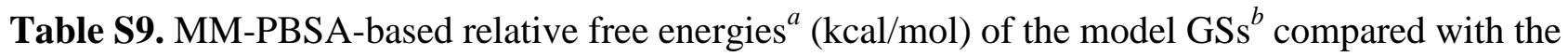
predictions based on the data by Cang et al.. ${ }^{1}$

\begin{tabular}{|c|c|c|c|c|c|c|}
\hline \multirow[t]{2}{*}{ GS } & \multirow{2}{*}{$\begin{array}{l}\text { Observed } \\
\text { number of } \\
5 \text { '- } \\
\text { hydrogen } \\
\text { bonds }{ }^{c} \text { in } \\
\text { the } \\
\text { simulations }\end{array}$} & \multirow{2}{*}{$\begin{array}{l}\text { Expected } \\
\text { number of } \\
5 \text { '-hydrogen } \\
\text { bonds }{ }^{c}\end{array}$} & \multicolumn{3}{|c|}{$\begin{array}{l}\text { Calculated relative free energy } \\
\text { (without the loops) }\end{array}$} & \multirow{2}{*}{$\begin{array}{l}\text { Predicted } \\
\text { relative free } \\
\text { energy (without } \\
\text { the loops) }\end{array}$} \\
\hline & & & bsc0 & $\operatorname{bsc} 0 \chi_{\mathrm{OL} 4}$ & $\operatorname{bsc} 0 \chi_{\mathrm{OL} 4} \varepsilon \xi_{\mathrm{OL} 1}$ & \\
\hline $\mathbf{0}$ & 2 & 2 & 0 & 0 & 0 & 0 \\
\hline 1 & 2 & 4 & -5 & -1 & -6 & -4 \\
\hline 2 & 2 & 2 & -2 & 14 & 4 & 19 \\
\hline 3 & 0 & 0 & 27 & 23 & 15 & 33 \\
\hline 4 & 0 & 2 & 12 & 31 & 11 & 29 \\
\hline 5 & 1 & 2 & 3 & 2 & -5 & 5 \\
\hline 6 & 0 & 0 & 18 & 48 & 11 & 33 \\
\hline 7 & 2 & 4 & -8 & -8 & $\begin{array}{c}-12 \\
\text { (30 -70 ns) }\end{array}$ & -4 \\
\hline
\end{tabular}

${ }^{a}$ The relative free energies of the GS were calculated with respect to GS $\mathbf{0}$ in the same force-field

${ }^{b}$ Simulations and MM-PBSA calculations were carried out with only the GSs of the models. The MM-PBSA calculations were carried out on 50-100 ns region of the trajectory, unless specified.

${ }^{c} 5$ '-OH - (G)N3 H-bonds at the 5'-end of the GSs.

The relative free energies of unstable GS arrangements are shown in italics in the Table. The relative free energy values were rounded-off to the nearest whole numbers. 
Table S10. MM-PBSA-based free energies ${ }^{a}(\mathrm{kcal} / \mathrm{mol})$ of the $\mathrm{GSs}^{b}$ of the models.

\begin{tabular}{|lllllllll|}
\hline GS & MM_ele & MM_vdw & MM_int PB_sur E_disper PB_cal & $G$ & $\Delta G$ \\
\hline bsc0 & & & & & & & & \\
\hline GS 0 & -1957 & -99 & 537 & 25 & -234 & -1027 & -2755 & 0 \\
\hline GS 1 & -1977 & -101 & 539 & 25 & -233 & -1013 & -2760 & -5 \\
\hline GS 2 & -1956 & -107 & 538 & 25 & -232 & -1025 & -2757 & -2 \\
\hline GS 3 & -1905 & -104 & 538 & 25 & -230 & -1052 & -2728 & 27 \\
\hline GS 4 & -1958 & -106 & 548 & 25 & -231 & -1021 & -2743 & 12 \\
\hline GS 5 & -1982 & -102 & 553 & 25 & -234 & -1012 & -2752 & 3 \\
\hline GS 6 & -1927 & -103 & 552 & 26 & -235 & -1050 & -2737 & 18 \\
\hline GS 7 & -1984 & -95 & 542 & 26 & -236 & -1016 & -2763 & -8 \\
\hline bsc0 $\chi_{\text {oL4 }}$ & & & & & & & & \\
\hline GS 0 & -1961 & -101 & 540 & 25 & -233 & -1029 & -2759 & 0 \\
\hline GS 1 & -1998 & -98 & 545 & 25 & -235 & -999 & -2760 & -1 \\
\hline GS 2 & -1970 & -105 & 547 & 25 & -230 & -1012 & -2745 & 14 \\
\hline GS 3 & -1917 & -104 & 542 & 26 & -232 & -1051 & -2736 & 23 \\
\hline GS 4 & -1914 & -106 & 547 & 25 & -229 & -1051 & -2728 & 31 \\
\hline GS 5 & -1969 & -101 & 546 & 25 & -235 & -1023 & -2757 & 2 \\
\hline GS 6 & -1921 & -110 & 553 & 25 & -226 & -1032 & -2711 & 48 \\
\hline GS 7 & -1997 & -99 & 544 & 25 & -235 & -1005 & -2767 & -8 \\
\hline bsc0 $\chi_{\text {oL4 } 4 \text { \& }}$ oL1 & & & & & & & \\
\hline GS 0 & -1960 & -101 & 545 & 25 & -233 & -1025 & -2749 & 0 \\
\hline GS 1 & -1991 & -98 & 542 & 25 & -233 & -1000 & -2755 & -6 \\
\hline GS 2 & -1974 & -102 & 547 & 25 & -232 & -1009 & -2745 & 4 \\
\hline GS 3 & -1925 & -103 & 545 & 25 & -232 & -1044 & -2734 & 15 \\
\hline GS 4 & -1978 & -102 & 553 & 25 & -233 & -1003 & -2738 & 11 \\
\hline GS 5 & -1968 & -101 & 548 & 25 & -235 & -1023 & -2754 & -5 \\
\hline GS 6 & -1946 & -103 & 549 & 25 & -233 & -1030 & -2738 & 11 \\
\hline GS 7 & -2002 & -99 & 546 & 25 & -235 & -996 & -2761 & -12 \\
\hline
\end{tabular}

${ }^{a}$ The individual terms in the Table are explained in the footnotes of the Table S3.

${ }^{b}$ Simulations and MM-PBSA were carried out only on the GSs of Htel topologies. The simulations were carried out in $0.15 \mathrm{M}$ excess $\mathrm{NaCl}$. 
Table S11. MM-GBSA-based free energies ( $\mathrm{kcal} / \mathrm{mol}$ ) of the antiparallel GQ models.

\begin{tabular}{|c|c|c|c|c|c|c|}
\hline \multirow[b]{2}{*}{ Model $^{a}$} & \multicolumn{2}{|l|}{ bsc0 } & \multicolumn{2}{|c|}{ bsc0 $\chi_{\mathrm{OL} 4}$} & \multicolumn{2}{|c|}{ bsc0 $\chi_{\mathrm{OL} 4} \varepsilon \zeta_{\mathrm{OL} 1}$} \\
\hline & G & $\Delta \boldsymbol{G}$ & G & $\Delta \boldsymbol{G}$ & G & $\Delta \mathbf{G}$ \\
\hline $\mathbf{0}$ & -2704 & 0 & -2705 & 0 & -2706 & 0 \\
\hline 1 & -2702 & 2 & -2707 & -2 & -2704 & 2 \\
\hline 2 & -2703 & 1 & -2690 & 15 & -2703 & 3 \\
\hline 3 & -2687 & 17 & -2699 & 6 & -2690 & 16 \\
\hline 4 & -2701 & 3 & -2693 & 12 & -2695 & 11 \\
\hline 5 & -2692 & 12 & -2695 & 10 & -2692 & 14 \\
\hline 6 & -2688 & 16 & -2684 & 21 & -2681 & 25 \\
\hline 7 & -2705 & -1 & -2704 & 1 & -2708 & -2 \\
\hline GS-Model $^{b}$ & G & $\Delta \mathbf{G}$ & $G$ & $\Delta \mathbf{G}$ & $G$ & $\Delta \mathbf{G}$ \\
\hline GS 0 & -2447 & 0 & -2449 & 0 & -2441 & 0 \\
\hline GS 1 & -2453 & -6 & -2440 & 9 & -2453 & -12 \\
\hline GS 2 & -2451 & -4 & -2443 & 6 & -2440 & 1 \\
\hline GS 3 & -2425 & 22 & -2440 & 9 & -2436 & 5 \\
\hline GS 4 & -2441 & 6 & -2430 & 19 & -2437 & 4 \\
\hline GS 5 & -2443 & 4 & -2447 & 2 & -2444 & -3 \\
\hline GS 6 & -2453 & -6 & -2430 & 19 & -2437 & 4 \\
\hline GS 7 & -2449 & -2 & -2451 & -2 & -2450 & -9 \\
\hline
\end{tabular}

${ }^{a}$ Simulations were carried out with complete GQs and subsequent MM-GBSA calculations were carried out only with the GSs.

${ }^{b}$ Simulations and MM-GBSA calculations were carried out only with the GSs of the models.

$G$ represents the energy of the model while $\Delta G$ represents relative free energy with respect to model 0. The simulations were carried out in $0.15 \mathrm{M}$ excess $\mathrm{NaCl}$. 


\section{REFERENCE}

(1) Cang, X.; Sponer, J.; Cheatham, T. E. Explaining the Varied Glycosidic

Conformational, G-tract Length and Sequence Preferences for Anti-parallel G-quadruplexes. Nucleic Acids Res. 2011, 39, 4499-4512. 